\title{
Forensic Gemmology in Forensic Practice
}

\author{
M. Kotrlý and I. Turková \\ Institute of Criminalistics Prague (ICP), POBox 62/KUP, Strojnicka 27, 17089 Praha 7, Czech Republic
}

At world market can be currently encountered a wide range of gemmological objects that are incorrectly declared, treated, or it concerns completely different materials that try to copy precious stone commodities more or less successfully. Materials in question are made using the latest technologies, however, only simple equipment is not sufficient to identify the true state of objects.

Optical polarised microscopy is the essential method for many materials, by means of which it is possible to determine an array of synthetic materials that are declared as natural, using research of mineral and technological inclusions, gas-liquid inclusions and inner structures. This method is also in most cases sufficient for authentication of moldavites (tektites) that are frequently replaced with glass. Chemical analysis fails in some materials because synthetic materials have identical composition with natural phase. Natural moldavites include lechatelierite inclusions that are not present in substitutes and that can be proved relatively well using optical microscope.

For some materials is b eneficial to use microspectrophotometry that can be conducive to ph ase differentiation having precisely defined colour. For example, these are so-called Bohemian Garnets and Bohemian Garnet Jewel, which have been and remain well known terms and are protected by the Office for industrial property as a designation of origin under numbers 80 "Bohemian Garnet" and 81 Bohemian Garnet jewellery". From a mineralogical standpoint, these are garnets with considerable prevalence of pyrope components, stable content of chromium and magnesium, originated from territorially very limited area and are typical of their dark red colour so-called "colour of pigeon blood". For initial quick orientation has proved it self very well in practice quantitative colour measurement. Since reproducible measurement of cut stones embedded in jewels is not entirely trivial, a few microspectrophotometric systems were tested, applying optical microscopes, direct macroscopic measurement with an entrance pupil of optic fibres zoomed in an object measured, as well measurement using integrating sphere "Labshere". Focused illumination related only to a part of the stone is significant with a view to minimise reflections. A range of spectrometers were tested - e.g. fy Avantes AvaSpec 2048, and other, Stellarnet - Black Comet CXR, Carl Zeiss MCS UV/NIR USB, etc. The results show that sensitivity of spectrometers is different in individual parts of the spectrum and for practice it is possible to compare only measurement made on identical device.

$\mathrm{SEM} / \mathrm{EDS} / \mathrm{WDS}$ and XRF/microXRF systems are used mostly for the study of chemical composition. In performing analyses of faceted stones fitted in jewels using SEM systems, in some cases can be found critical both signal homogeneity in area at nonstandard operating distances and also position of an analyzed surface towards SEM de tector with regard to a pplication of ZAF corrections. Experiments carried out have shown that differences in resul ts of quantitative analysis of homogenous material performed from spots having various geometric positions towards detector can amount to tens of percents. Obviously, all these factors can be emended, but an operator mustn't approach the analysis in a way "one touch of button yields a full result".

For calibration of quantitative analysis are indispensable standards. Unfortunately, when testing these standards it turns out that some of them are not in too high quality even from renowned providers. For 
example, rests of polishing powders were ascertained lodged in the surface of soft materials that can distort considerably subsequent use of standard, nonhomogeneity, etc.

Treatment of gemmological objects consisting in filling microfissures with a high refractive index are relatively frequent. It concerns modifications that are not forbidden, but they have to be declared and enclosed with a stone, which does not often happen. These alterations could be well documented through flat mapping using SEM/EDS, possibly microXRF.

To help in cases regarding questionable classification of pearls (particularly differentiation so-called natural versus cultivated pearls that differ considerably by price, but their surface composition and microscopic surface characteristics could be the same) were tested possibilities of X-ray backlight. X-ray backlight method has been developed that can be used also on XRF table devises with transmission detector.

Experiments that have been conducted have proved that for differentiation of modern sophisticated imitations of gemmological objects is not sufficient only simple magnifying glass, which so me gemmologist still do not fully admit. In addition to above mentioned techniques are employed also other methods - FTIR, XRD, microXRD, etc.

Fakes with substitution of natural stones for their imitations are unfortunately very frequent. Last year, Czech Trade Inspection carried out checks in three towns in the Czech Republic, executed inspection purchases and in the course of subsequent expert examinations were in 100\% (of cases) detected stones that were incorrectly declared.

\section{References:}

[1] Newbury D., Ritchie N.: Scanning microscopies 2012, SPIE, Vol. 8378, 2012, pp. 837803-1 - 12.

[2] McClure S. F.: GEMS \& GEMOLOGY, Vol. 42, No. 1, 2006, pp. 22 - 34

[3] Kotrly M., Turkova I: Scanning microscopies 2012, SPIE, Vol. 8378, 2012, pp. 83780Y-1 - 8

[4] Acknowledgements - microanalytical methods at ICP were supported by projects:

RN19961997008, RN19982000005, RN20012003007, RN20052005001, VD20062008B10,

VD20072010B15, VG20102015065, VF20112015016. 